\title{
Assessment of pertussis vaccination strategies using a mathematical model of disease transmission
}

\author{
Pablo Pesco, B.S. ${ }^{a}$, Paula Bergero, M.D. ${ }^{a}$, Gabriel Fabricius, M.D. ${ }^{a}$, and \\ Daniela Hozbor, M.D.
}

\begin{abstract}
Pertussis or whooping cough is a vaccinepreventable respiratory disease that has reemerged in the past decades. A higher morbidity and mortality has been recorded in infants, although cases have also been reported in adolescents and adults. The epidemiological scenario for this condition has urged to review and implement new strategies aimed at improving its control.

However, many of these strategies havenotbeen investigated in depth so as to be established as universal. In this context, mathematical models of disease transmission are useful decisionmaking tools.

Using a mathematical model of pertussis, this study assessed the possible impact of the different control measures on the most vulnerable population (0-1 year old infants). In particular, the analysis focused on the impact of including a booster vaccination at 11 years old, the effect of improving the coverage provided by primary doses, and the reduction of any delay in their administration.

The assessment also estimated the effect of immunizing pregnant women.

Results show that including a booster dose at 11 years reduces the incidence of pertussis by $3 \%$ in infants younger than 1 year old. In addition, administering primary doses in compliance with the schedule (with no delays) reduces pertussis incidence by $16 \%$. Increasing coverage from $80 \%$ to $95 \%$ results in a significantly decreased incidence in the vulnerable population (38\%). If the percentage of immunized pregnant women reaches $50 \%$, the reduction of the most severe infant cases could be more than $43 \%$ (0-2 monthold infants).

Key words: pertussis, Bordetella pertussis, pertussis vaccine, immunization, booster, model.
\end{abstract}

http:/ /dx.doi.org/10.5546/aap.2013.373

\section{INTRODUCTION}

Pertussis (whooping cough) is a highly contagious acute respiratory disease caused by Bordetella pertussis. Most severe cases affect infants younger than 1 year old who have not been immunized or with incomplete immunization schedules with a resulting lack of protection. ${ }^{1-3}$
The most significant complications are hospitalization, bronchopneumonia, seizures, acute encephalopathy, permanent brain injury, and death. ${ }^{4}$

The disease is transmitted personto-person. Patients are often most infective during the first three weeks after the onset of symptoms. This period is significantly shorter (5 days) with an adequate antibiotic therapy.

Whole-cell or acellular vaccines are used for pertussis control. The type of vaccine used depends, among other factors, on the age of the population. Whole-cell vaccines are not recommended for children older than 7 years old. Acellular vaccines have different formulations targeted for the pediatric population and the adolescent and adult population, basically with a different dose of immunogens. The mass use of vaccines significantly reduced pertussis morbidity and mortality, and led to a change in its epidemiology, thus shifting the burden of disease away from children and increasing the relative burden among infants and in the adolescent and adult population..$^{5-7}$ In the vaccination era, adolescents and adults appear to be the main source of infection for young children. ${ }^{8}$ In the pre-vaccination era, given the high circulation of this bacterium in the population, adults used to acquire natural boosters more frequently due to their recurrent exposure to pertussis cases. Mothers who acquired immunity this way would transfer the protection to their children, resulting in a lower incidence of severe cases among infants. Most severe cases usually occurred in 3-6 year old children.

In the past years, several countries 
have recorded a sustained increase in the number of cases. ${ }^{5,9-11}$

In Argentina an increase was detected as of 2002, which continues to date. For example, during 2012, 8670 suspected cases of pertussis were reported to the National Surveillance System (SIVILA); out of them, 6911 were in infants younger than 1 year old. Most cases were found in the most densely populated provinces of Argentina (Buenos Aires, Córdoba and Santa Fe). Of the total number of cases, 1117 were confirmed by the laboratory. During 2010, 4981 cases were reported, with 828 laboratory-confirmed cases. Once again, most cases occurred in infants younger than 1 year old (4217). The high rate of infant cases is not unexpected because pertussis is most severe in this age group.

Several causes may have contributed to the increase in the number of cases detected: a strengthened surveillance, the implementation of new diagnostic methods, a relatively low vaccine efficacy, the short duration of vaccinegenerated immunity, and the adaptability of the causative agent to the immunity conferred by the vaccines. ${ }^{5,12,13}$

Regardless of the causes, the significant increase in the number of cases has prompted health systems of different countries to review and implement new strategies aimed at improving pertussis control, especially in the most vulnerable population: infants younger than 1 year old. These strategies include cocooning, immunizing healthcare personnel who come into contact with children, immunizing pregnant women, and adding a booster for adolescents. ${ }^{14-16}$ Given the recent implementation of some of these strategies, it is yet not possible to implement a universal strategy. In this context, mathematical models that simulate pertussis transmission can be a decision-making support tool. ${ }^{17-20}$ This article presents our model predictions regarding the impact on the incidence of pertussis among the most vulnerable population using some of the strategies put into practice in Argentina, and the effect of improving the coverage provided by primary doses and compliance with the national immunization schedule (NIS).

\section{POPULATION AND METHODS}

Data related to the age of administration of vaccine doses included in this study are retrospective and were provided by the Immunization Center of Hospital Elena de la Serna Montes de Oca, located in downtown La
Plata in the Province of Buenos Aires. The study period was between January 2005 and May 2012, and included 29845 records of pertussis vaccinations in children between 0 and 12 months old. Infants older than 12 months old, those living in a different province, and those whose vaccination or age data were confounding or incomplete were excluded.

In Argentina, the immunization schedule includes three primary doses at 2, 4 and 6 months old (DPT3), one dose at 18 months old, and boosters at the time of starting primary education and at 11 years old. ${ }^{21}$ Except for the 11 year old dose (Tdap11), which is an acellular formulation, the other doses are a combination of whole-cell vaccines and other immunogens.

According to the WHO, the immunization coverage provided during the first year of life to infants in Argentina varied between 87\% and $94 \%$ in the $2006-2011$ period, with the minimum coverage attained in 2009..$^{22}$ When DPT3 vaccination coverage is broken down by department, some departments show coverage values under $80 \%{ }^{23}$

\section{Model}

This study used a deterministic mathematical model developed by our group for simulating pertussis transmission. ${ }^{24}$ The model includes a population structured by age, immune status, and individuals' infectivity. The model considers a population stratified into 9 epidemiological classes and 30 age groups. The dynamics of pertussis transmission was simulated by transferring individuals from one class to the other at different specific rates, as shown in Figure 1.

In this model, when individuals are born (if mothers have not transferred them antibodies), they are categorized in the $S$ class (susceptible), where they will remain unless: a) they get infected and become infective as a result of being in contact with an infected individual and become I1 symptomatic infection class, or b) they acquire partial immunity with the first vaccine dose, when they become P1 AI class (partial acquired immunity). When individuals receive consecutive vaccine doses (indicated in Figure 1 with dotted lines), they can go through classes of increasing immunity and, eventually, reach the CAl class (complete acquired immunity), which means a complete immunity acquired through vaccination. We considered that only one fraction of individuals who receive one vaccine dose actually pass to the next immunity class (vaccine 
efficacy, VE), because it is known that pertussis vaccines are not $100 \%$ effective (see Annex in electronic version). Individuals in class P1 and PAl can acquire the infection (and pass to class 12 or 13 , respectively) and develop the disease, but with fewer symptoms, therefore, they are less infective. The rate at which susceptible individuals (or those with partial immunity) in a given age group acquire the infection is called force of infection $\mathrm{A}$. The calculation of A considers contact patterns between individuals of different age groups, fractions of infected individuals in each group, and their respective infectivity.

Based on the resolution of the differential equations describing the model dynamics, it is possible to estimate the specific incidence by age in each age group $i$ : Inc ${ }_{1 \mathrm{i}}=\lambda_{\mathrm{i}} \mathrm{S}_{\mathrm{i}}$ (cases with complete symptoms), $\mathrm{Inc}_{2 \mathrm{i}}=\lambda_{\mathrm{i}} \mathrm{P}_{\mathrm{AI}}^{1}$ (cases with partial symptoms) where $S_{i}$ and $\mathrm{P}_{\mathrm{AI}}^{1}$ are the populations in classes $\mathrm{S}$ and $\mathrm{PAl}$ respectively for the individuals in the age group $i$.

We will specifically focus on the incidence $\left(\operatorname{lnc}_{1}+\operatorname{lnc}_{2}\right)$ in the $0-1$ year old age range.

\section{Establishing parameters}

The model requires parameters with information on the disease characteristics and its transmission, and on vaccination. Some of these parameters are hard to determine, such as immunity duration, which has not yet been agreed upon by experts. Other parameters are not homogenous across the population, such as vaccination coverage and specific contact patterns by age. In order to include such variability, we have considered different scenarios where parameter values are combined so as to encompass a wide range of possible situations.

Such processes allow us to explore the sensitivity of results when parameters change, and identify as robust those parameters that are independent from the scenario being considered. This article presents the results obtained using the CP1A-MDI scenario described above. ${ }^{24}$ The CP1AMDI scenario sets the parameters for the contact among individuals patterns based on the force of infection values estimated from epidemiological data obtained during the pre-vaccination era,

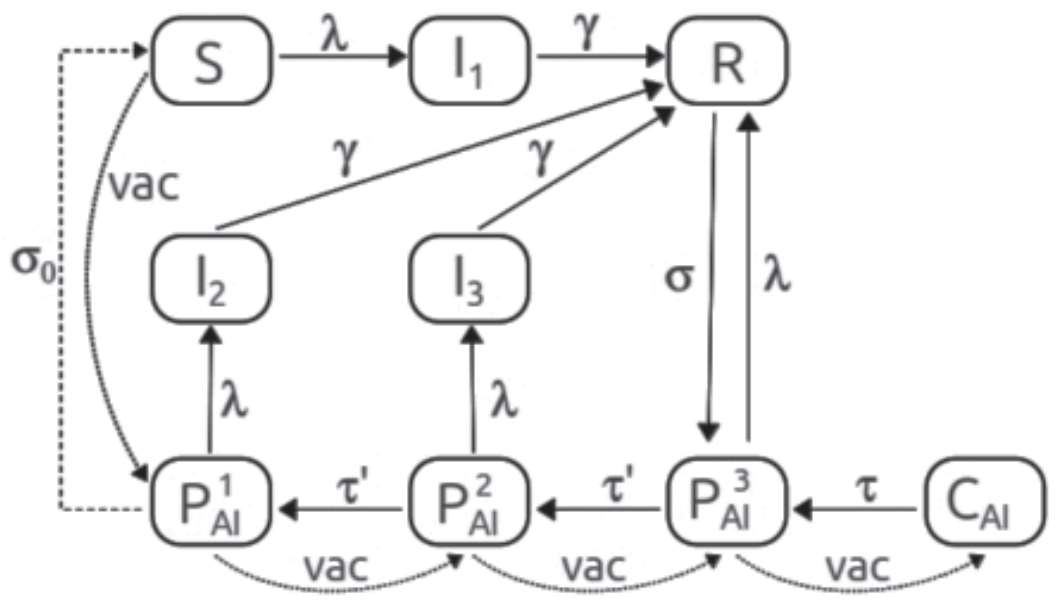

In this model, individuals are born in class S (if they have not received antibodies from their mothers), then they acquire the infection and become class $\mathrm{I}_{1}$, or are partially immunized with the first vaccine dose and become class $\mathrm{P}_{\mathrm{Ar}}^{1}$. With the successive vaccine doses, individuals go through classes $\mathrm{P}_{\mathrm{AI}}^{1} \rightarrow \mathrm{P}_{\mathrm{AI}}^{2} \rightarrow \mathrm{P}_{\mathrm{AI}}^{3} \rightarrow \mathrm{C}_{\mathrm{AI}}$, attaining complete immunity with the last one. Individuals in classes $\mathrm{P}_{\mathrm{AI}}^{1}$ and $\mathrm{P}_{\mathrm{AI}}^{2}$ can become infected and become classes $\mathrm{I}_{2}$ or $\mathrm{I}_{3}$, respectively. Infection disappears in a period of $1 / \gamma=21$ days. After this period, individuals in class $\mathrm{I}_{1}, \mathrm{I}_{2}$ $\mathrm{o} \mathrm{I}_{3}$ recover and become class $\mathrm{R}$. The model considers that immunity, whether acquired through infection or vaccination, does not last for life and is gradually lost. Individuals in any of the partial or complete immunity classes go through $\mathrm{P}_{\mathrm{AI}}$ classes by reducing their acquired immunity level at the given rates $\left(\sigma, \tau, \tau^{\prime}\right)$. Over a very long period $\left(1 / \sigma_{0}\right)$, they can become totally susceptible $\left(\mathrm{P}_{\mathrm{AI}}^{1} \rightarrow \mathrm{S}\right)$. 
assuming a mean immunity duration of 15 years for infection-generated immunity, and of 6 years for vaccine-generated immunity.

\section{RESULTS}

\section{Effects of delayed primary vaccination}

The administration of the vaccine at a moment beyond the window established by the NIS increases the risk of disease. In Argentina, vaccination coverage has been improved; yet, delays in the administration of recommended doses still occur. ${ }^{25,26}$ Based on the studied records, it has been detected that a high proportion of DPT3 doses are not given at the time specified in the NIS (Figure 2). Forty-nine percent of children received the first vaccine dose at least one week later, and some had delays of up to 40 days.

In relation to the second and third doses, delays of at least one week were recorded in $67 \%$ and $74 \%$ of children, respectively. To evaluate the impact of vaccination delays on the incidence of pertussis in the 0-1 year old group, a comparison was made between children in this age group using two types of primary dose coverage ( $80 \%$ and $95 \%)$ and two vaccination profiles (A, no delay; B, delayed) (Figure 3). Results show that when there are no delays in the dose administration, the incidence of pertussis in infants younger than 1 year old is reduced when compared to the incidence estimated with a delayed administration. Thus, for example, giving primary doses with no delays and with a $95 \%$ coverage leads to a reduction of $16 \%$ in the incidence of pertussis in infants younger than 1 year old.

\section{Effects of improving primary vaccination coverage}

Since DPT3 coverage is heterogeneous across the country, calculations were made taking into account the two types of coverage reported: $95 \%$ and $80 \%$.

Increasing the coverage from $80 \%$ to $95 \%$ for primary doses leads to a reduction in the incidence of pertussis among the most vulnerable individuals of $38 \%$ when doses are administered on time (Figure 3, panel A) and of $36 \%$ when the administration is delayed (Figure 3, panel B).

Results refer to the sum of the Inc (cases $_{1}$ with complete symptoms) and $\mathrm{Inc}_{2}$ (cases with moderate infection) incidences. With a 95\% coverage, Inc accounts for $43 \%$ of the sum Inc C $_{1}+$ Inc $_{2}$. With an $80 \%$ coverage, Inc 1 accounts for $53 \%$ of such sum; therefore, improving the coverage would result in an additional advantage for the reduction of the most severe cases.

FIGURE 2. Distribution by age at the time of administration of the three primary doses of the pertussis vaccine

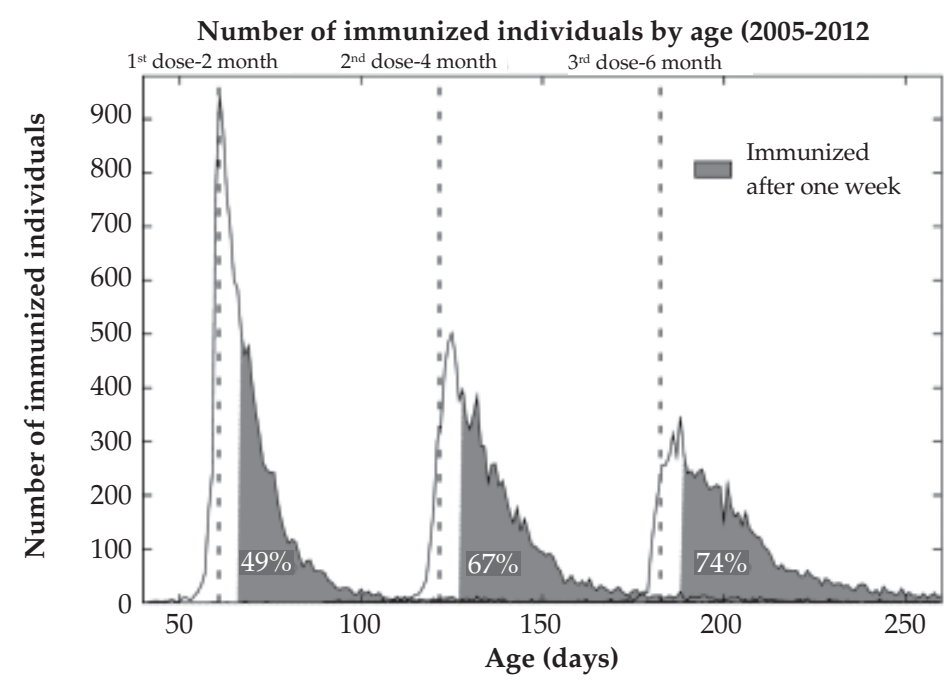

Data were provided by the Immunization Center of Hospital Elena de la Serna de Montes de Oca for the 2005-2012 period. Yearly profiles were prepared with 3468, 3677, 2433, 4418, 4687, 4449, 5219 and 1494 records, respectively, and show little variation from the average profile. The vertical dotted lines indicate the NIS recommended age for the administration of each pertussis vaccine primary dose. In addition, the figure shows the percentage of immunized individuals with a delay of more than one week. 


\section{Effects of introducing a dose at 11 years old}

The main objective of the Tdap11 booster is to reduce the incidence of pertussis in adolescents and also in infants younger than 1 year old. Although adolescent immunization appears to imply a decreased source of infection for these infants, there is no evidence to support such hypothesis.

In our model, introducing the Tdap11 booster caused a significant reduction in the incidence among adolescents (37\%). However, the incidence reduction in the most vulnerable population (Figure 3) was less than 3\%. For calculation purposes, the Tdap11 coverage was considered to be $72 \%$ (data from 2011) with an $80 \%$ primary dose coverage, and $85 \%$ with a $95 \%$ primary dose coverage. Likewise, an improvement in coverage from $85 \%$ to $95 \%$ for the dose at 18 months old resulted in a $10 \%$ reduction in the $18-36$ monthold group, but it only decreased the incidence of pertussis by $1 \%$ in infants younger than 1 year old.

\section{Effects of immunization in mothers}

Our model allows to consider the effects of maternal transfer of immunity to infants with the addition of a class composed of individuals with maternally-derived antibodies. ${ }^{23}$ This way, assuming that antibodies last 2.5 years following the infection, a fraction of mothers in the $\mathrm{R}$ class would result in newborn infants protected for their first two months of life. If individuals in this class acquired the infection, they would develop the disease with fewer symptoms $\left(\mathrm{I}_{2}\right)$.

When considering such effect in this study, the model recorded a reduction of approximately $6 \%$ in the Inc1 incidence. With such modification in the model, it is possible to make an approximate estimation of the effect of immunity transfer by immunized mothers on their infants. For example, if $50 \%$ of mothers transfer immunity to their babies, a $43 \%$ reduction in the occurrence of severe cases is observed in infants younger than 2 months old $\left(\mathrm{Inc}_{1}\right)$. These results highlight the importance of this strategy aimed at protecting

FIGURE 3. Comparison of pertussis incidences in the 0-1 year old age range when doses are administered on time $(A)$ and with the delays shown in Figure 2 (B)

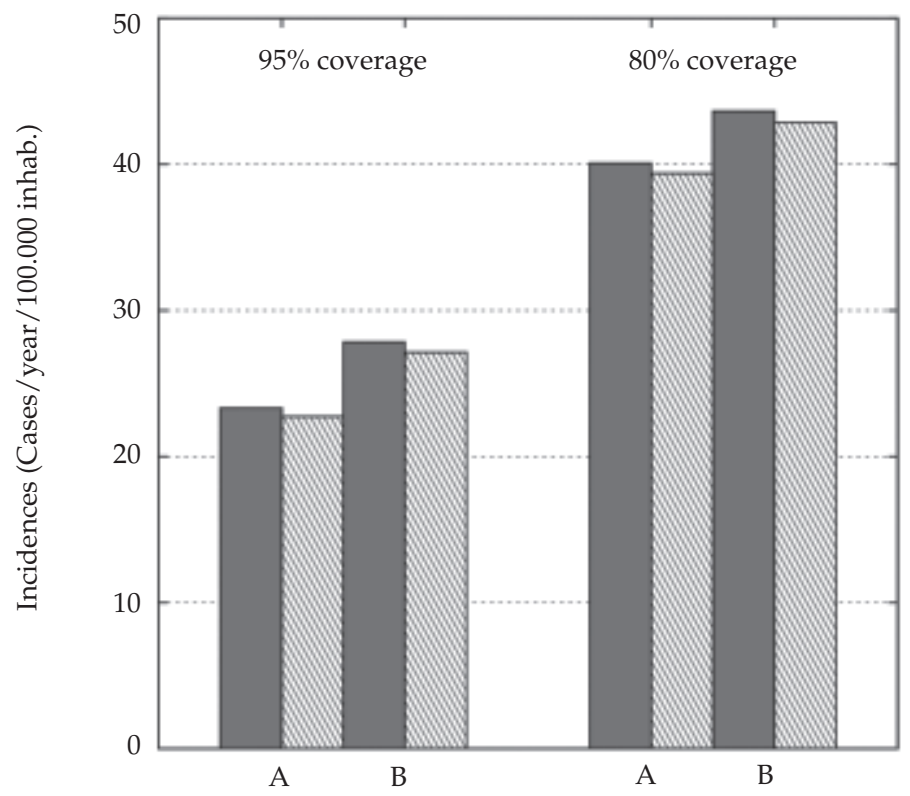

Results for the $95 \%$ and $80 \%$ coverage are compared. The stripped bars show the additional effect of introducing a booster at 11 years old. 
a population who cannot be immunized using vaccines. For this estimation, a 95\% coverage for the three primary doses and a delay profile were used.

\section{DISCUSSION}

The epidemiological situation of pertussis in Argentina and other countries prompts a review of the strategies implemented to control the disease. The analyses made in this study show the benefits of attaining a $95 \%$ coverage with the three primary doses and administering them at the time recommended in the NIS, avoiding delays whenever possible. It is worth remembering that delay profiles used are representative of the population in downtown La Plata; however, it is expected that delays are even more prolonged in suburban areas.

Dayan, et al. ${ }^{26}$ analyzed the reasons for vaccination delays and noticed that some, like a lack of vaccines or ignorance regarding the immunization schedule, can be prevented with adequate measures; while other delays, such as being ill or convalescent at the time of vaccination, cannot be solved.

In relation to the booster dose administered at 18 months old, improving coverage only reduces the incidence of pertussis by $1 \%$ in infants younger than 1 year old. Introducing vaccination at 11 years old also causes a small reduction in the incidence in the 0-1 year old group. Such prediction is consistent with what other authors have reported, who use different models and parameters. ${ }^{17,27-30}$

This may be due, at least partially, to a relatively low burden of disease among adolescents as a source of infection for infants. ${ }^{30-33}$ Adults, particularly parents, appear to be the main source of infection for infants. ${ }^{34}$ It is expected that the estimated incidence reduction would be even higher if mothers were immunized with an adequate coverage (above the 50\% used in this study).

Even though it is evidently advantageous to compare the effectiveness of control measures against a disease using a mathematical model as the one used in this study, it is not aimed at predicting absolute incidence values because they can vary depending on the parameters used (see Annex). However, the sensitivity analysis shows that the model reliably reproduces the relative burden of the different studied effects and predicts which measures would be more effective.

\section{CONCLUSIONS}

According to our model, improving the primary dose coverage above $95 \%$ and complying with the national immunization schedule with no delays could have a significant impact on the reduction of pertussis incidence in infants younger than 1 year old (38\%). Administering the vaccine to pregnant women is also presented as a relevant strategy because considering only the effect of protection acquired through antibody transfer, immunizing $50 \%$ of mothers reduces the number of most severe cases by $43 \%$ in newborn infants younger than 2 months old.

The strategy that seems to have the lesser effect on infants is administering a booster dose at 11 years old, which only caused a small reduction (less than 3\%) in the incidence of pertussis in the most vulnerable group.

All adjustments made to current control measures, which could have a clear impact on the incidence of pertussis in infants, should be accompanied by new studies on the epidemiology and causes of pertussis, and by the design of new strategies that allow a more effective control of this disease, which today is a burden on public health.

\section{Acknowledgments}

We would like to thank Mario Arrúa. M.D. for his selfless help to obtain the data used in this study. The study was funded by the National Agency of Scientific and Technological Promotion (Agencia Nacional de Promoción Científica y Tecnológica, ANPCyT) and the National Scientific and Technical Research Council (Consejo Nacional de Investigaciones Científicas y Técnicas, CONICET). Pablo Pesco is a CONICET grant holder, Paula Bergero and Gabriel Fabricius are in taking part in the CONICET science degree, and Daniela Hozbor is taking part in the CICBA science degree.

\section{BIBLIOGRAPHY}

1. WHO. Pertussis: immunization surveillance, assessment and monitoring. [Accessed on: June 8, 2008]. Available at http://www.who.int/immunization_monitoring/ diseases/pertussis/en/index.html.

2. Dauer CC. Reported whooping cough morbidity and mortality in the United States. Pub Health Rep 1943;58:66177.

3. MattooS,CherryJD.Molecularpathogenesis, epidemiology, and clinical manifestations of respiratory infections due to Bordetella pertussis and other Bordetella subspecies. Clin Microbiol Rev 2005;18:326-82.

4. CDC. Pertussis complications. [Accessed on: December 
27, 2012]. Available at http://www.cdc.gov/pertussis/ about/complications.html.

5. Cherry J. Epidemic pertussis in 2012--the resurgence of a vaccine-preventable disease. N Engl J Med 2012;367:785-7.

6. Anon. Pertussis-United States, 1997-2000. Morbidity and Mortality Weekly Report 2002;51:73-6.

7. Riva Posee CA, Miceli INP. Evolución de la coqueluche en la Argentina a finales del siglo XX. Medicina 2005;65:7-16.

8. Wiley KE, Zuo Y, Macartney KK, McIntyre PB. Sources of pertussis infection in young infants: A review of key evidence informing targeting of the cocoon strategy. Vaccine 2012 in press doi: 10.1016/j.vaccine.2012.11.052.

9. de Melker HE, Coyin-van Spaendonck, Rumke HC, van Wijngaarden JK, et al. Pertussis in The Netherlands: an outbreak despite high levels of immunization with wholecell vaccine. Emer Infect Dis 1997;3:175-8.

10. Hozbor D, Mooi F, Flores D, Weltman G, et al. Pertussis epidemiology in Argentina: trends over 2004-2007. J Infect 2009;59:225-31.

11. Base de datos del Ministerio de Salud de Argentina. [Accessed on:December 27,2012]. Availableat:http://msal. gov.ar/htm/site/pdf/epidemiologia/boletinsemanal/ BoletinIntegradoDeVigilanciaVersionVF_SE34.pdf.

12. Wendelboe AM, Van Rie A, SalmasoS, Englund J. Duration of immunity against pertussis after natural infection or vaccination. Pediatr Infect Dis Jour 2005;24:S58-61.

13. Bart MJ, van Gent M, van der Heide HG, Boekhorst J, et al. Comparative genomics of prevaccination and modern Bordetella pertussis strains. BMC Genomics 2010;11:627.

14. CDC. Updated recommendations for use of tetanus toxoid, reduced diphtheria toxoid and acellular pertussis vaccine (Tdap) in pregnant women and persons who have or anticipate having close contact with an infant aged $<12$ months. Advisory Committee on Immunization Practices (ACIP), 2011. Morbidity and Mortality Weekly Reports 2011;60:1424-26.

15. Forsyth KD, Wirsing von Konig, Tan T, Caro J, et al. Prevention of pertussis: recommendations derived from the second Global Pertussis Initiative roundtable meeting. Vaccine 2007;25:2634-42.

16. Brooks DA, Clover R. Pertussis Infection in the United States: Role for Vaccination of Adolescents and Adults. J Am Board Fam Med 2006;19:603-11.

17. Hethcote HW. Simulations of pertussis epidemiology in the United States: effects of adult booster vaccinations. Mathemat Biosc 1999;158:47-73.

18. van Boven M, de Melker HE, Schellekens JFP, Kretzschmar M. Amodel based evaluation of the 1996-7 pertussis epidemic in the Netherlands. Epidemiol Infect 2001;127:73-85.

19. Rohani P, Zhong X, King AA. Contact network structure explains the changing epidemiology of pertussis. Science 2010;330:982-5.

20. Lavine JS, King AA, Bjornstad ON. Natural immune boosting in pertussis dynamics and the potential for longterm vaccine failure. PNAS 2011;108:7259-64.
21. Ministerio de salud (Argentina). Calendario nacional de vacunación. [Accessed on: December 27, 2012]. Available at http://www.msal.gov.ar/index.php/ component/ content/article/46/184-calendario-nacionaldevacunacion-2012.

22. OMS.WHO-UNICEF estimates of immunization coverage: Argentina. [Accessed on: December 27, 2012]. Available at: http://apps.who.int/immunization monitoring/en/ globalsummary/timeseries/TSWUcoverageByCountry. cfm? country=ARG.

23. Base de datos del Ministerio de Salud (Argentina). [Accessed on: December 27, 2012]. Available at: http:/ / msal. gov.ar/htm/site/pdf/AnexoVII_PAI-03-09.pdf.

24. Fabricius G, Bergero PE, Ormazabal ME, Maltz AL, et al. Modelling pertussis transmission to evaluate the effectiveness of an adolescent booster in Argentina. Epidemiol Infect 2013;141(4):718-34.

24. Hethcote HW. An age-structured model for pertussis transmission. Mathem Biosc 1997;145:89-136.

25. Dayan GH, Shaw KM, Baughman AL, Orlellana LC, et al. Assessment of delay in age-appropriate vaccination using survival analysis. Am J Epidemiol 2006;163:561-70.

26. Gentile A, Bakir J, Firpo V, Caruso M, et al. Esquemas atrasados en la vacunación y oportunidades perdidas de vacunación en niños de hasta 24 meses: estudio multicéntrico. Arch Argent Pediatr 2011;103(3):219-25.

27. Quinn HE, McIntyre PB. The impact of the adolescent pertussis immunization, 2004-2009: lessons from Australia. Bull World Health Organization 2011;89:666-74.

28. Skoff TH, Cohn AC, Clark TA, Messonnier NE, et al. EarlyImpact of the USTdap vaccination program on pertussis trends. Arch Pediat Adol Med 2012;166:344-9.

29. Lavine JS, Bjornstad ON, de Blasio BF, Storsaeter J. Shortlived immunity against pertussis, age-specific routes of transmission, and the utility of a teenage booster vaccine. Vaccine 2012;30:544-51.

30. Rozenbaum MH, De Vries R, LE HH, Postma MJ. Modelling the impact of extended vaccination strategies on the epidemiology of pertussis. Epidemiol Infect 2011. doi:10.1017/S0950268811002354.

31. Jardine A, Conaty SJ, Lowbridge C, Staff M, et al. Who gives pertussis to infants? Source of infection for laboratory confirmed cases less than 12 months of age during an epidemic, Sydney, 2009. Comm Dis Intel 2010;34:116-21.

32. Bisgard KM, Pascual FB, Ehresmann KR, Miller CA, et al. Infant pertussis: who was the source? Ped Infect Dis J 2004;23:985-9.

33. Wendelboe AM, Njamkepo E, Bourillon A, Floret DD, et al. Transmission of Bordetella pertussis to young infants. Pediat Infect Dis J 2007;26:293-9.

34. Anderson R, May R. Infectious Diseases of Humans: Dynamics and Control. Oxford: Oxford UniversityPress; 1991.

35. Anderson R, May R. Infectious diseases of humans: dynamics and control. Oxford: Oxford University Press; 1991. 


\section{Annex. Parameters used in the model}

Produced following a type I mortality, 35 which consists in assuming that all individuals live exactly up to the same age, in this case, 75 years old. We have considered that the infection lasts a mean period of 21 days. To assess the efficacy of each vaccine dose, we considered that $\mathrm{VE}=0.9$, as in reference 25 . This implies that, in our model, 1-2 years after the third vaccine dose, it is predicted that $86-95 \%$ of the population would be protected against a severe form of pertussis, and $63-81 \%$ would be protected against acquiring a moderate form of the infection. In terms of the coverage provided by the different doses, this study included two cases: $95 \%$ and $80 \%$. With the $95 \%$ coverage, it is assumed that $95 \%$ of the population receives the corresponding doses at 2, 4 and 6 months old, and at 6 years old, but a smaller proportion $(85 \%)$ receives the dose at 18 months old. With an $80 \%$ coverage, the same criteria were applied, and it is assumed that $70 \%$ of the population receives the dose at 18 months old.

In our model, we considered that individuals in classes I 2 and I 3 have a lower infectivity than those in class $\mathrm{I}_{1}$. This is taken into account through the 1 and 2 factors, which are lower than 1 , and thus put into perspective their contribution to the force of infection of those with a lower infectivity.

$\lambda_{i}=\sum_{j} \beta_{i j} I_{j}^{*} ; I_{j}^{*}=I_{1 j}+\rho_{1} I_{2 j}+\rho_{2} I_{3 j}$

We used $1=0.5$ and $2=0.25$, as in reference 25 .

\section{Infective contact and immunity duration parameters for the CP1A-MDI scenario}

TABLE 1. Contact rates, $i j$ (in 1/year), among age group $i$ (row) and age group $j$ (column) for the CP1A scenario. The matrix is symmetrical, so some elements have been left out

\begin{tabular}{|c|c|c|c|c|c|c|c|c|c|}
\hline Age & $0-4 \mathrm{~m}$ & $4 \mathrm{~m}-1 \mathrm{a}$ & $1-3 a$ & $3-5^{a}$ & $5-10 a$ & $10-15 a$ & $15-35 a$ & $35-55 a$ & $55-75 a$ \\
\hline $0-4 m$ & 0.02 & 0.02 & 0.02 & 0.02 & 0.02 & 0.02 & 0.12 & 0.12 & 0.12 \\
\hline $4 m-1 a$ & & 0.12 & 0.12 & 0.12 & 0.12 & 0.12 & 0.23 & 0.23 & 0.23 \\
\hline $1-3 a$ & & & 0.23 & 0.23 & 0.23 & 0.23 & 0.23 & 0.23 & 0.23 \\
\hline $3-5 a$ & & & & 0.73 & 0.73 & 0.23 & 0.23 & 0.23 & 0.23 \\
\hline $5-10 a$ & & & & & 2.47 & 0.95 & 0.23 & 0.23 & 0.23 \\
\hline $10-15 a$ & & & & & & 0.95 & 0.54 & 0.16 & 0.0 \\
\hline $15-35 a$ & & & & & & & 0.54 & 0.16 & 0.0 \\
\hline $35-55 a$ & & & & & & & & 0.16 & 0.0 \\
\hline $55-75 a$ & & & & & & & & & 0.0 \\
\hline
\end{tabular}

\section{Duration of immunity against pertussis}

TABLE 2. Mean immunity duration (MID) parameters

\begin{tabular}{cccccc}
\hline$t_{\mathrm{N}}$ & $\mathrm{t}_{\mathrm{v}}$ & $1 / \sigma$ & $1 / \tau$ & $1 / \tau^{\prime}$ & $1 / \sigma_{0}$ \\
15 & 6 & 11 & 2 & 2 & 100 \\
\hline
\end{tabular}

$t_{N}$ and $t_{v}$ are the durations of naturally-acquired immunity (through infection) or artificially-acquired immunity (through vaccination), respectively. Rates $\sigma, \tau$ and $\tau^{\prime}$ are chosen to obtain $t_{N}=1 / \sigma+2 / \tau^{\prime}, t_{v}=1 / \tau+2 / \tau^{\prime}$. The pace at which immunized individuals become completely susceptible is controlled by the rate, $\sigma_{0}$. All values are expressed in years. 


\section{SENSITIVITY ANALYSIS}

In reference [24] the transmission of pertussis was studied using different parameters representative of varied epidemiological scenarios. All such scenarios reproduce the known characteristics of pertussis epidemiology and are therefore "likely" scenarios. Table 3 shows how the main results presented in this article (for the CP1A-MDI scenario) are modified when a model with other scenario parameters is used. Although values of the incidences vary depending on the different scenarios, it has been observed that all conclusions drawn regarding the studied vaccination strategies are maintained for all scenarios, and are therefore considered robust conclusions.

\section{Estimated incidence for different scenarios and strategies}

TABLE 3. Total incidences (Inc $1+$ Inc 2) in infants younger than 1 year old for different studied scenarios and strategies

\begin{tabular}{lccccccc}
\hline Scenari & $\begin{array}{c}\text { COB 95-B } \\
\mathbf{( 1 )}\end{array}$ & COB 95-A & $\begin{array}{c}\text { Percent change } \\
\text { from (1) }\end{array}$ & $\begin{array}{c}\text { COB } \\
\text { 95-B +11a }\end{array}$ & $\begin{array}{c}\text { Percent change } \\
\text { from (1) }\end{array}$ & $\begin{array}{c}\text { COB } \\
\text { 80-B }\end{array}$ & $\begin{array}{c}\text { Percent change } \\
\text { from (1) }\end{array}$ \\
\hline CP1A-LDI & $\mathbf{2 5 . 5}$ & 21.3 & -16.2 & 24.5 & -3.7 & 41.1 & 61.3 \\
CP1A-MDI & $\mathbf{2 7 . 5}$ & 23.3 & -15.4 & 27.2 & -1.2 & 43.6 & 58.4 \\
CP1A-SDI & 35.0 & 30.0 & -14.5 & 34.4 & -1.8 & 52.2 & 48.9 \\
CP1B-LDI & 33.0 & 27.4 & -17.2 & 32.7 & -1.0 & 49.5 & 49.8 \\
CP1B-MDI & $\mathbf{3 5 . 1}$ & 29.1 & -17.1 & 34.8 & -0.9 & 52.3 & 48.7 \\
CP1B-SDI & $\mathbf{4 3 . 6}$ & 36.8 & -15.6 & 43.2 & -1.0 & 63.6 & 45.9 \\
CP2-LDI & $\mathbf{2 1 . 6}$ & 19.1 & -11.5 & 20.8 & -3.5 & 35.2 & 63.2 \\
CP2-MDI & $\mathbf{2 8 . 2}$ & 25.1 & -10.8 & 27.3 & -2.9 & 42.7 & 51.8 \\
CP2-SDI & $\mathbf{4 6 . 4}$ & 42.1 & -9.2 & 45.6 & -1.7 & 69.8 & 50.5 \\
Average & $\mathbf{3 2 . 9}$ & 28.3 & -14.1 & 32.3 & -2.0 & 50.0 & 53.2 \\
DCM & $\mathbf{8 . 2}$ & 7.4 & 2.9 & 8.3 & 1.1 & 11.0 & 6.2 \\
\hline
\end{tabular}

The column in bold represents the case used as reference to estimate the percentage changes for each strategy (COB 95-B= 95\% coverage in the primary pertussis series with the delay profile shown in Figure 2, COB 95- $\mathrm{A}=95 \%$ coverage in the primary pertussis series with no delays, $\mathrm{COB} 80-\mathrm{B}=80 \%$ coverage in the primary pertussis series with the delay profile shown in Figure 2, COB 95-B + Vac 11a $=95 \%$ coverage in the primary pertussis series with the delay profile shown in Figure 2 plus the administration of a vaccine at 11 years old with an $85 \%$ coverage and no delay). In addition, the table shows the average and mean squared deviation (MSD) for each situation.

You can request more details on the parameters included in the model and the sensitivity analysis to the authors by e-mail. 